www.jmscr.igmpublication.org

Impact Factor 5.84

Index Copernicus Value: 83.27

ISSN (e)-2347-176x ISSN (p) 2455-0450

crossref DOI: _https://dx.doi.org/10.18535/jmscr/v5i3.214

Journal Of Medical Science And Clinical Research

IGM Publication

An Official Publication of IGM Publication

\title{
Outcome of Treatment in Uterine Sarcoma: Fifteen Years Experience at A Regional Cancer Centre
}

\author{
Author \\ Dr Ujwala Prakash Wakpaijan \\ Cancer Institute, Adyar, Chennai
}

\begin{abstract}
Objective: To evaluate the pattern of recurrence and survival of uterine sarcoma patients treated at regional cancer centre.

Background: Uterine sarcomas are a rare but extremely aggressive subtype of uterine malignancies. The prognosis is poor and the role of adjuvant treatment has not yet been established.

Methods: We conducted a retrospective analysis of the medical records of patients diagnosed with uterine sarcomas who were treated in our Institute from 1998 to 2013. The tumour characteristics, stage of disease, treatment modalities, histological types, follow up and survival were studied. Patients with metastatic disease at diagnosis were excluded from the analysis.

Results: A total of 38 patients were selected for the study. Among these, 16 patients (42\%) were operated elsewhere and the remaining 22 patients (58\%) underwent surgery in our institution. The proportion of patients with Stage I, II and III disease according to the FIGO staging were $73.5 \%, 21 \%$ and $5.5 \%$ respectively. The histological types included Carcinosarcomas (47.5\%), liomyosarcomas (26\%), endometrial stromal sarcoma high grade (7\%), endometrial stromal sarcoma low grade (13\%) and undifferentiated tumours (7\%). Among the patients who underwent surgery in our institution, $68 \%$ patients received adjuvant radiotherapy to the pelvis, $14 \%$ received adjuvant radiotherapy and sequential chemotherapy, $14 \%$ received only chemotherapy and one patient received no adjuvant treatment. At last follow-up, 27 patients (76.3\%) had died of the disease and 9 (23.7\%) are alive \& healthy. The median disease free survival was 17 months (range 5 to 39 months). And median overall survival was 39 months range (6 to 60 months). Distant metastasis outside the abdominal cavity occurred in 17 patients, whereas peritoneal and pelvic recurrence was seen in 10 and 3 patients respectively.

Conclusion: Surgery remains the main stay of treatment for uterine sarcoma regardless of histological type of tumour. Most important part of surgery is to resect the tumour with all margins free of tumour. The prognostic factors for improved survival are stage I, II disease. Adequate surgery with good tumour free margins in the pelvis, Adjuvant with radiotherapy by external beam RT to pelvis had prevented the local recurrences but did not improve overall survival. Prospective clinical trials with adjuvant modalities treatment are needed to improve survival.

Keywords: uterine sarcoma, endometrial stromal sarcoma, Carcinosarcomas, survival.
\end{abstract}

\section{Introduction}

Uterine sarcoma accounts for3- $5 \%$ of all uterine malignancies ${ }^{(1)}$. This tumor has been considered as highly aggressive, showing a poor prognosis ${ }^{(2)}$.
It mainly comprises the pathological subgroups of Carcinosarcomas [formerly known as malignant mixed Mullein tumor, (MMMT)] as the most common histology (50\%), leiomyosarcoma (30\%) 
and the remaining consists of endometrial stromal sarcoma (ESS) (10\%). Each group has its own risk factors and clinical manifestations, treatment, response, prognosis and specific genetic aberrations ${ }^{(3)}$.

Most of patients present with abnormal bleeding per vaginum and diagnosis is difficult prior to Surgery. Radiological modalities do not add more to differentiate the tumour. In early stage surgery is the main stay of treatment the surgery includes total hysterectomy and bilateral salphingoopherectomy, pelvic nodal involvement is documented only $2-3 \%$, in early stage disease. In liomyo sarcoma. Role of staging laparatomy with bilateral pelvic nodal dissection and omentectomy is not indicated .ovarian preservation in early stage liomyosarcomas may be considered. But in advanced cases lymphadenectomy may be needed. In case of Carcinosarcomas with disease at extrauterine sites cytoreductive surgery can be done. Morcellation of the tumour or uterus in total during laparoscopic assisted hysterectomy increases the rate of the abdomino-pelvic dissemination causing an iatrogenic advanced stage disease. So removal of tumour in piece meal or Morcellation is contraindicated, it has worse survival. Histological type is an independent prognostic variable for survival in uterine sarcomas. Low-grade ESS has the best clinical outcome, whereas leiomyosarcoma has the poorest one. It is noteworthy that, when adjusting for stage and mitotic count, leiomyosarcoma has a significantly worse prognosis than Carcinosarcomas. Uterine Carcinosarcomas are biphasic malignant tumor consisting of epithelial and mesenchymal components arising from a common epithelial cel, they are mostly seen in patients on prolonged tamoxifen therapy and radiotherapy, and usually these patients are in advanced stage of disease at presentation ${ }^{(4,5)}$.

Adjuvant treatment is completely dependent of histopathological type of tumour. Treatment of recurrence of disease is dealt with the site and type of recurrence. If single site recurrence which can be resected by surgery, surgery is done ${ }^{(6)}$.
There are no specific clinical trials for management of recurrent disease this indicates the rarity of disease. A prospective phase II trial testing the combination chemotherapy of gemcitabine and docetaxel followed by doxorubicin in stage I/II disease reported promising results regarding activity (2 year PFS: $78 \%$ ) $(7,8)$. The combination of carboplatin and pegylated liposomal doxorubicin also demonstrated activity in another phase II trial [Harter et al. 2011).This study is retrospective analysis of case records of patients who were treated at this hospital and followed up from 1998 to 2013.

WHO classification their histopathologic classification was revised by the World Health Organization (WHO) in 2014 the current World Health Organization recognizes categories of endometrial stromal tumor: Endometrial stromal nodule (ESN), Low-grade endometrial stromal sarcoma (LG-ESS), High-grade endometrial stromal sarcoma (HG-ESS), and Undifferentiated (Tab: 1).

Liomyosarcomas, endometrial stromal sarcomas are diagnosed are mostly after surgery on histopathogy. There are no phase III randomised trials for definitive adjuvant therapy.For advanced Carcinosarcomas Adjuvant chemotherapy with lipodox, gemcitabine. Isofinamide,cisplatin had been tried, There is no Role Of Radiotherapy in liomyosarcomas In endometrial stromal sarcomas, who are receptor positive progesterones like megastral acetate, Letrozole anastrazole had been used.

\section{Material and Methods}

We conducted a retrospective analysis of the medical records of patients diagnosed with uterine sarcomas who were treated in our Institute from 1998 to 2013. The tumour characteristics, stage of disease, treatment modalities, histological types, follow up and survival were studied. The case records of patients who were registered under uterine malignancies were selected. Patients with uterine sarcoma were segregated; Patients with metastatic disease at diagnosis were excluded 
from the analysis. The tumour characteristics, stage at the time of diagnosis, radiological findings treatment modalities chemotherapy and radiotherapy offered to patient follow up and survival were noted. Most of patient had died due to disease, so retrieval of recurrence site, most were on progressive disease. Condition of patient at the terminal of stage of disease was not recorded in the case sheet.

\section{Results}

A total of 38 patients were selected for the study. Most of the patient was operated outside as there was no preoperative diagnosis, and referred to our institute for further treatment among these, 16 patients $(42 \%)$ were operated elsewhere and the remaining 22 patients $(58 \%)$ were diagnosed and underwent surgery in our institution. The patients who were treated before 2003 were staged according to the old FIGO staging and patient registered after 2004 were staged as per new who staging. The proportion of patients with Stage I, II and III disease according to the FIGO staging were $73.5 \%, 21 \%$ and $5.5 \%$ respectively. The histological types included Carcinosarcomas $(47.5 \%)$, liomyosarcomas (26\%), endometrial stromal sarcoma high grade $(7 \%)$, endometrial stromal sarcoma low grade (13\%) and undifferentiated tumours $(7 \%)$.

Among the patients who underwent surgery in our institution, $68 \%$ patients received adjuvant radiotherapy to the pelvis, $14 \%$ received adjuvant radiotherapy and sequential chemotherapy, $14 \%$ received only chemotherapy, chemotherapy drugs were doxorubicin and gemcitabine combination and one patient received no adjuvant treatment. At last follow-up, 27 patients (76.3\%) had died of the disease and $9(23.7 \%)$ are alive \& healthy. The median disease free survival was 17 months (range 5 to 39 months). And median overall survival was 39 months range (6 to 60 months). Distant metastasis outside the abdominal cavity occurred in 17 patients, whereas peritoneal and pelvic recurrence was seen in 10 and 3 patients respectively.
Table: 1

\begin{tabular}{|l|l|}
\hline \multicolumn{2}{|l|}{ Classification of uterine sarcoma(FIGO) 2009} \\
\hline $\begin{array}{l}\text { Uterine Sarcomas (Liomyosarcomas, Endometrial Stromal } \\
\text { Sarcoma and Adenosarcoma) }\end{array}$ \\
\hline IA & Tumor limited to uterus $<5 \mathrm{~cm}$ \\
\hline IB & Tumor limited to uterus $>5 \mathrm{~cm}$ \\
\hline IIA & Tumor extends to the pelvis, adnexal involvement \\
\hline IIB & Tumor extends to extra-uterine pelvic tissue \\
\hline IIIA & Tumor invades abdominal tissues, one site \\
\hline IIIB & More than one site \\
\hline IIIC & $\begin{array}{l}\text { Metastasis to pelvic and/or para-aortic lymph } \\
\text { nodes }\end{array}$ \\
\hline IVA & Tumor invades bladder and/or rectum \\
\hline IVB & Distant metastasis \\
\hline Adenosarcoma Stage I Differs from Other Uterine Sarcomas \\
\hline IA & Tumor limited to endometrium/endocervix \\
\hline IB & Invasion to $<1 / 2$ myometrium \\
\hline IC & Invasion to $>1 / 2$ myometrium \\
\hline
\end{tabular}

Table 2

\begin{tabular}{|l|c|c|}
\hline Charecteristics & Number & Percentage (\%) \\
\hline Carcinosarcomas & 18 & 47.5 \\
\hline Liomyosarcomas & 8 & 26 \\
\hline Endometrial stromal sarcoma & 6 & 13.5 \\
\hline Endometrial stromal sarcoma & 3 & 7 \\
\hline Undifferentiated sarcoma & 3 & 7 \\
\hline
\end{tabular}

Table: 3

\begin{tabular}{|l|c|c|}
\hline Charecteristics & Number & Percentage (\%) \\
\hline Operated elsewhere & 16 & 42 \\
\hline Operated at institute & 22 & 58 \\
\hline Stage I & 27 & 73.5 \\
\hline Stage II & 8 & 21 \\
\hline Stage III & 3 & 5.5 \\
\hline
\end{tabular}

\section{Discussion}

Uterine sarcomas are very rare tumour accounts for 3-5 \% of all uterine malignancies. Due to biological aggressive nature of tumour, the diagnosis often made retrospectively after surgery in most of patients. The common symptoms on presentation are abnormal uterine bleeding, Post menopausal bleeding per vaginum, abdominal lump. Abdominal distension endometrial biopsy and fractional curettage is not contributory ,as tumour are mesenchymal, patients presenting in advanced stage may had ascities ,extrauterine disease, and raised level of ca 125,with lung and liver metastasis ${ }^{(5,9)}$. The median age at presentation age was 62 yrs (+- 5yrs) the presenting symptom were abnormal uterine 
bleeding .abnormal discharge per magnum followed by abdominal discomfort and abdominal mass. Patients who were operated outside and then referred to our institute had total abdominal hysterectomy with bilateral salphingo-opherectomy.

Patient who underwent surgery at our hospital all had frozen section; patient with liomyosarcomas underwent total abdominal hysterectomy and bilateralsalphingo-opherectomy. Patient with Carcinosarcomas. undifferentiated sarcomas underwent staging laparatomy like endometrial carcinoma. With cytoreductive surgery of extrauterine disease, one patient was deemed inoperable due to extensive peritoneal disease. Ned one patient underwent uereteric reimplantation, as tumour was infiltrating the uerete.

Postoperative histopathological review was carried out for pathological staging. Patient with myometrial infiltration of tumour more than half were given adjuvant radiotherapy, Lymphovascular space invasion, very close resected margin to tumour or one of the margin positive for tumour. Endo metrial stromal sarcoma of low grade was followed up. The $68 \%$ patient received adjuvant postoperative radiotherapy to pelvis 40 to 50 grey, followed by vaginal brachytherapy. patient with ascities fluid cytology and peritoneal washing positive for tumour mostly Carcinosarcomas and undifferentiated sarcomas received adjuvant chemotherapy doxorubicin and gemcitabine 6 cycles. All this patients were followed up every 3 monthly for first three years and the every 6 monthly for next 2 years, at the end of one year of treatment asymptomatic patients were investigated with chest x-ray. Ultrasonographic of abdomen.

In symptomatic patients they were evaluated as per symptoms. the median age of recurrence was 13 months range 5 to 39 months, The site of recurrence were lungs, liver peritoneal cavity And par aortic nodes, three patients had liver and lung metastasis simultaneously. Patient with recurrence of with endometrial stromal sarcoma were treated with Letrozole or oral progesterone megastral acetate. At last follow-up, 27 patients (76.3\%) had died of the disease and $9(23.7 \%)$ are alive \& healthy. The median disease free survival was 17 months (range 5 to 39 months). And median overall survival was 39 months range (6 to 60 months). Distant metastasis outside the abdominal cavity occurred in 17 patients, whereas peritoneal and pelvic recurrence was seen in 10 and $3 \%$.

\section{Conclusion}

Surgery remains the main stay of treatment for uterine sarcoma regardless of histological type of tumour. Most important part of surgery is to resect the tumour with all margins free of tumour. The prognostic factors for improved survival are stage I, II disease. Adequate surgery with good tumour free margins in the pelvis, Adjuvant with radiotherapy by external beam RT to pelvis had prevented the local recurrences but did not improve overall survival. Prospective clinical trials with adjuvant modalitie treatment are needed to improve survival.

\section{References}

1. Toro, J. R. et al. Incidence patterns of soft tissue sarcomas, regardless of primary site, in the surveillance, epidemiology and end results program, 1978-2001: An analysis of 26,758 cases. International journal of cancer119, 2922-2930 (2006).

2. Bocker, W. [WHO classification of breast tumors and tumors of the female genital organs: pathology and genetics]. Verhandlungen der Deutschen Gesellschaft fur Pathologie86, 116-119 (2002).

3. D'Angelo, E., Spagnoli, L. G. \&Prat, J. Comparative clinicopathologic and immunohistochemical analysis of uterine sarcomas diagnosed using the World Health Organization classification system. Human pathology40, 1571-1585 (2009).

4. D'Angelo, E. \&Prat, J. Uterine sarcomas: a review. Gynecologic oncology116, 131139 (2010). 
5. Wolfson, A. H. et al. A multivariate analysis of clinicopathologic factors for predicting outcome in uterine sarcomas. Gynecologic oncology52, 56-62 (1994).

6. Hensley, M. L. et al. Adjuvant therapy for high-grade, uterus-limited leiomyosarcoma: results of a phase 2 trial (SARC 005). Cancer119, 1555-1561 (2013).

7. Giuntoli, R. L., 2nd et al. Retrospective review of 208 patients with leiomyosarcoma of the uterus: prognostic indicators, surgical management, and adjuvant therapy. Gynecologic oncology89, 460-469 (2003).

8. Li, N. et al. Treatment options in stage I endometrial stromal sarcoma: a retrospective analysis of 53 cases. Gynecologiconcology108, 306-311 (2008). 